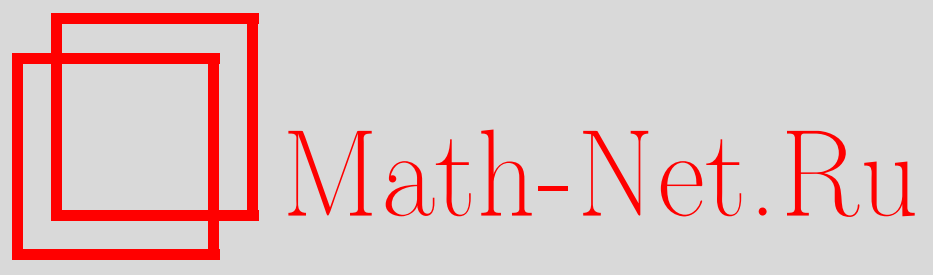

Общероссийский математический портал

Р. И. Григорчук, Эргодические теоремы для действий свободной группы и свободной полугруппы, Матем. заметки, 1999, том 65, выпуск 5, 779-783 


\section{DOI: https://doi.org/10.4213/mzm1109}

Использование Общероссийского математического портала Math-Net.Ru подразумевает, что вы прочитали и согласны с пользовательским соглашением http://www.mathnet.ru/rus/agreement

Параметры загрузки:

IP : 18.208 .226 .222

26 апреля 2023 г., $17: 23: 32$ 


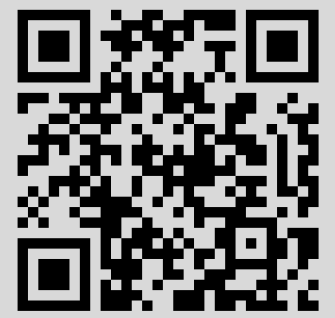




\section{ЭРГОДИЧЕСКИЕ ТЕОРЕМЫ ДЛЯ ДЕЙСТВЙ̆ СВОБОДНОЙ ГРУППЫ И СВОБОДНОЙ ПОЛУГРУППЫ}

\section{Р.И. Григорчук}

1. Введение. Подобно тому как теорема Вейля о равномерном распределении на окружности предшествовала появлению эргодических теорем Биркгофа и фон Неймана, теорема Арнольда и Крылова [1] о равномерном распределении на сфере предшествовала эргодическим теоремам для действий некоммутативных групп преобразований.

Вначале были доказаны: статистическая эргодическая теорема для действий аменабельных групп, индивидуальная эргодическая теорема для действий аменабельных групп, удовлетворяющих условию Кальдерона [2], и статистическая эргодическая теорема для унитарных представлений свободной группы [3].

В 1987 году автор анонсировал индивидуальную эргодическую теорему для действий свободной группы [4], а в 1994 году аналогичньй результат был переоткрыт в [5]. При этом был определен новый тип усреднения в конечно порожденных группах: рассмотрение чезаровских средних последовательности усреднений по сферическим подмножествам.

В этой заметке мы анонсируем несколько утверждений, носящих характер индивидуальной и статистической эргодических теорем для действий свободной группы и свободной полугруппы. Эти результаты докладывались на конференции по теории случайных блужданий и теории потенциала, проходившей в Кортона (Италия) в июне 1997 года. 
Основным новшеством в сформулированных ниже утверждениях является предположение о стационарности меры, а не более сильное условие ее инвариантности, обычно налагаемое в формулировках эргодических теорем. Наличие эргодических теорем в предположении стационарности меры является важным обстоятельством, что продиктовано текущими задачами. Например, стационарные меры возникают при рассмотрении границ Пуассона конечно порожденных групп, а также при исследовании статистических свойств фрактальных множеств. Существенным обстоятельством является также то, что инвариантная мера при действии неаменабельной группы на компакте обычно не существует, в то время как стационарная мера существует.

2. Индивидуальная эргодическая теорема для действия свободной полугруппы. Пусть $X$ - пространство с сигма-алгеброй измеримых множеств, $\bar{T}=\left\{T_{1}, \ldots, T_{m}\right\}$ - набор измеримых преобразований пространства $X, P=\left\{p_{1}, \ldots, p_{m}\right\}$ - распределение вероятностей. Назовем марковским оператором на функииях оператор $M$, действующий на функции по формуле:

$$
(M f)(x)=\sum_{i=1}^{m} p_{i} f\left(T_{i} x\right) .
$$

Аналогично определяется марковский оператор на мерах:

$$
M \mu=\sum_{i=1}^{m} p_{i} T_{i} \mu,
$$

где $(T \mu)(A)=\mu\left(T^{-1} A\right)$ для преобразования $T$ и измеримого подмножества $A \subset X$.

Мера $\mu$ назьвается $P$-стационарной, если $M \mu=\mu$. Очевидно, что мера, инвариантная относительно каждого из преобразований $T_{i}, 1 \leq i \leq m$, является стационарной.

Породив преобразованиями $T_{i}, 1 \leq i \leq m$, полугруппу преобразований, получим действие на $X$ (вообще говоря, неточное) свободной полугруппы $G=S F_{m}$ с $m$ порождающими (единица предполагается принадлежащей $G$ ). Элементы полугруппы $F S_{m}=\left\langle T_{1}, \ldots, T_{m}\right\rangle$ отождествляются со словами над алффавитом $\left\{T_{1}, \ldots, T_{m}\right\}$. Пусть $|g|$ - длина элемента $g \in F S_{m}$. Имеется $m^{n}$ элементов длины $n$, составляющих "сфферу" радиуса $n$.

Определим последовательность распределений $\left\{P_{n}\right\}_{n=1}^{\infty}$ на $G: P_{n}(g)=0$, если $|g| \neq n$, и $P_{n}(g)=p_{i_{1}} p_{i_{2}} \ldots p_{i_{n}}$, если $g=T_{i_{1}} T_{i_{2}} \ldots T_{i_{n}}$. При этом $P_{1}$ совпадает с исходным распределением $P$.

Для произвольной функции $f$ на $X$ определим средние по сферам

$$
M_{n} f=\sum_{|g|=n} P_{n}(g) \cdot(g f)
$$

и чезаровские средние

$$
C_{n} f=\frac{1}{n} \sum_{i=0}^{n-1} M_{i} f
$$

(заметим, что $M_{0}=1, M_{1}=M$ и $(g f)(x)=f(g x)$ ). Для равномерного распределения $P=$ $\{1 / m, \ldots, 1 / m\}$ средние (1) имеют вид:

$$
M_{n} f=\frac{1}{m^{n}} \sum_{|g|=n} g f .
$$

Tеорема 1. Пусть свободная полугруппа $G=F S_{m}$ с $m$ порождающими действует на пространстве с мерой $(X, \mu)$ и мера $\mu$ P-стационарна.

Тогда для любого $p, 1 \leq p<+\infty$, и произвольной функиии $f \in L_{p}(X, \mu)$ средние (2) сходятся $\mu$-почти непрерывно $к$ некоторой $M$-инвариантной функиии $\tilde{f} \in L_{p}(X, \mu)$, причем если $\mu(X)<\infty$, то выполнено равенство

$$
\int_{X} f(x) d \mu(x)=\int_{X} \tilde{f}(x) d \mu(x) .
$$

Также во всех случаях имеет место сходимость средних (2) $\kappa \tilde{f}$ по норме и функиия $\tilde{f}$ является $G$-инвариантной. 
3. Индивидуальные и статистические теоремы для действий свободной группы. Пусть $G=F_{m}$-свободная группа с $m$ свободными порождающими $T_{1}, \ldots, T_{m}$, действующая измеримьми преобразованиями на пространстве $X$ (а следовательно, действующая на функции и на меры на $X$ ) или изометриями банахова пространства $E$. Во избежание громоздких обозначений будем обозначать через $g x, g f, g \mu, g h$ образ точки $x \in X$, функции $f$ меры $\mu$ или вектора $y \in E$ под действием элемента $g \in G$, при этом $(g f)(x)=f\left(g^{-1} x\right),(g \mu)(A)=\mu\left(g^{-1} A\right)$.

Через $|g|$ обозначим длину $g$ относительно системы порождающих (т.е. длину записи элемента $g$ несократимым словом в алфавите $\left.\left\{T_{1}, \ldots, T_{m}, T_{1}^{-1}, \ldots, T_{m}^{-1}\right\}\right)$. Заметим, что имеется $2 m \times$ $(2 m-1)^{n-1}$ элементов длины $n$, составляющих "сферу" радиуса $n$.

Мера $\mu$ на $X$ называется стационарной, если

$$
\mu=\frac{1}{2 m} \sum_{\varepsilon= \pm 1} \sum_{i=1}^{m} T_{i}^{\varepsilon} \mu .
$$

По аналогии с полугрупповым случаем определим операторы усреднения по сферам и соответствуюшие им чезаровские средние:

$$
\begin{aligned}
M_{n} & =\frac{1}{2 m(2 m-1)^{n-1}} \sum_{|g|=n} g, \\
C_{n} & =\frac{1}{n} \sum_{i=0}^{n-1} M_{i} .
\end{aligned}
$$

Теорема 2. Пусть свободная әруппа $G=F_{m}$ с $m \geq 2$ образующими действует на пространстве $(X, \mu)$ и мера $\mu$ - стационарна.

Тогда гильбертово пространство $H=L_{2}(X, \mu)$ допускает разлохение $H=H_{-1} \oplus$ $H_{0} \oplus H_{1}$, где $H_{1}$ - подпространство $G$-инвариантных функций, подпространство $H_{-1}$ определяется соотношением

$$
H_{-1}=\{f \in H: M f=-f\},
$$

а на подпространстве $H_{0}$ последовательность операторов $M_{n}$ сильно сходится к нулевому оператору.

Теорема 3. Пусть свободная группа $G=F_{m}$ с $m \geq 1$ образующ,ими действует изометриями в рефлексивном банаховом пространстве $E$.

Тогда для любого $x \in E$ средние $C_{n} x$ сходятся $\kappa$ некоторому $M$-инвариантному вектору $y \in E$. Если $E$ строго выпукло, то у является $G$-инвариантным.

Переходим к формулировке индивидуальной эргодической теоремы.

Теорема 4. Пусть свободная әруппа $G=F_{m}$ c $m \geq 1$ образующими действует на пространстве $(X, \mu)$ и мера $\mu$ стачионарна.

Тогда имеют место все утвержсдения теоремы 1.

4. О доказательствах. В доказательстве теоремы 2 используется теорема о существовании унитарного степенного продолжения у произвольного сжатия в гильбертовом пространстве.

Доказывая теорему 3, мы применяем теорему Иосиды-Какутани-Рисса-Лорча к матричному оператору

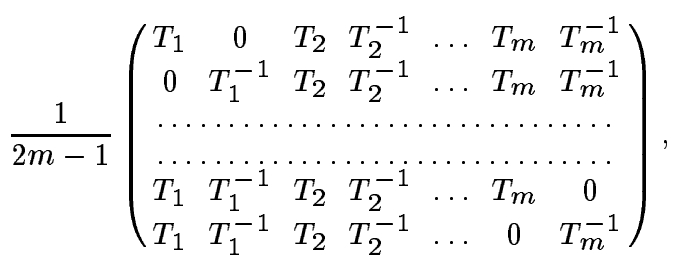


действующему в пространстве $E \oplus \cdots \oplus E(2 m$ слагаемых $)$.

При доказательстве индивидуальных эргодических теорем 1,4 применяется теорема Биркгофа и ее усиления для сжимающих операторов в форме теорем Данфорда-Шварца или Чакона и Акоглу. Редукция к классическим теоремам основана на конструкции скрещенного произведения динамической системы, порожденной несколькими преобразованиями на подходящий сдвиг Бернулли. Так, например, при доказательстве теоремы 1 используется скрещенное произведение $(Q, X \times$ $\left.\Omega_{m}, \mu \times \nu\right), Q(x, \omega)=\left(T_{\omega} x, \sigma \omega\right)$, динамической системы $\left(T_{1}, \ldots, T_{m}, X ; \mu\right)$ на сдвиг Бернулли $\left(\sigma, \Omega_{m}\right)$, где $\Omega_{m}=\left\{T_{1}, \ldots, T_{m}\right\}^{\mathbb{N}}, \nu$ - мера Бернулли, определенная распределением $T_{\omega}$, а $T_{\omega}$ совпадает с преобразованием $T_{i}$, если последовательность $\omega$ начинается с символа $T_{i}$. При этом, в качестве вспомогательных, фигурируют следующие два утверждения.

Лемма 1. Мера $\boldsymbol{\mu}$ является $P$-стачионарной тогда и только тогда, когда мера $\mu \times \nu$ $Q$-инвариантна.

ЛЕмма 2. Динамическая система $\left(T_{1}, \ldots, T_{m}, X ; \mu\right)$ эргодична в том и только том случае, если преобразование $Q$ эргодично.

При доказательстве теоремы 4 используется тот же прием, однако вместо пространства $\Omega_{m}$ рассматривается пространство $\Omega_{m}^{*}$, состоящее из свободно несократимых последовательностей символов алфавита $\left\{T_{1}, \ldots, T_{m}, T_{1}^{-1}, \ldots, T_{m}^{-1}\right\}$, снабженное равномерной мерой, т.е. мерой, определенной на цилиндрических множествах соотношением

$$
\mu\left(\overline{T_{i_{1}}^{\varepsilon_{1}} \ldots T_{i_{n}}^{\varepsilon_{n}}}\right)=\frac{1}{2 m(2 m-1)^{n-1}} .
$$

5. Энтропия системы некоммутирующих преобразований. Пусть $\left(T_{1}, \ldots, T_{m}\right.$, $X ; \mu)$ - динамическая система, порожденная преобразованиями $T_{1}, \ldots, T_{m}$ пространства $X$ (и тем самьм определено действие свободной полугруппы $\left.G=F S_{m}=\left\langle T_{1}, \ldots, T_{m}\right\rangle\right)$, причем мера $\mu$ является $P$-стационарной относительн некоторого распределения вероятностей $P$. Если $W-$ слово над алфавитом $\left\{T_{1}, \ldots, T_{m}\right\}$, определяющее элемент полугруппы $G$, а $\xi$-разбиение пространства $X$, то через $\xi_{W}$ обозначим разбиение $\bigvee_{U<W} T_{U}^{-1} \xi$, где $U$ пробегает множество начал слова $W$ и $T_{U}=T_{i_{1}} \ldots T_{i_{k}}$, если $U=T_{i_{1}} \ldots T_{i_{k}}$.

Пусть $H(\xi)$ обозначает энтропию разбиения $\xi$, т.е.

$$
H(\xi)=-\sum_{i=1}^{l} \mu\left(A_{i}\right) \log \mu\left(A_{i}\right)
$$

где $\left\{A_{i}\right\}_{i=1}^{l}$ есть множество атомов разбиения $\xi$.

Определим энтропию набора $\bar{T}=\left\{T_{1}, \ldots, T_{m}\right\}$ относительно разбиения $\xi$ как

$$
h(\bar{T}, \xi)=\varlimsup_{n \rightarrow \infty} \frac{1}{n} \sum_{|W|=n} P_{n}(W) H\left(\xi_{W}\right),
$$

где суммирование ведется по всем словам длины $n$, а $P_{n}$-последовательность распределений, определенная в п. 2. В случае равномерного распределения соотношение (3) приобретает вид

$$
h(\bar{T}, \xi)=\varlimsup_{n \rightarrow \infty} \frac{1}{n} \sum_{|W|=n} H\left(\xi_{W}\right) .
$$

Наконец, определим энтропию набора $\bar{T}$ равенством $h(\bar{T})=\sup _{\xi} h(\bar{T}, \xi)$.

Tеорема 5. Имеет место соотношение $h(\bar{T})=h(Q)-h(\sigma)$, где $Q-$ скрещенное произведение динамической системы $(\bar{T}, X, \mu)$ на сдвиг Бернулли $\left(\sigma, \Omega_{m}, \nu\right)$, определенное $в$ n. $4, a h(\sigma)=-\sum p_{i} \log p_{i}-$ энтропия сдвига Бернулли.

Отметим, что понятие топологической энтропии набора преобразований $\bar{T}$ компактного метрического пространства введено Буфетовым в [6], где также получена формула, аналогичная соотношению из теоремы 5 . 


\section{СПИСОК ЦИТИРОВАННОЙ ЛИТЕРАТУРЫ}

1. Арнольд В. И., Крылов А. Л. // Докл. АН СССР. 1962. Т. 4. № 1. С. 1-5. 2. Темпельман А. А. // Докл. АН СССР. 1967. Т. 176. № 2. С. 190-193. 3. Guivarc'h Y. // Comptes Rendus Acad. Sci. 1969. V. 268. Р. 1020. 4. Григорчук Р. И. Индивидуальная эргодическая теорема для действий свободных групп // Тезисы докладов XII школы по теории операторов в функциональных пространствах. Ч. 1. Тамбов, 1987. С. 57. 5. Nevo A., Stein E. M. // Acta Math. 1994. V. 173. P. 135-154. 6. Bufetov A. Topological entropy of free semigroup actions and skew-product transformations // Dynamical and Control Systems. To appear..

Математический институт им. В. А. Стеклова РАН 